\title{
Gas Turbine Tubular Coilpipes Regenerators Optimized According to the Minimum Mass Criterion \\ Gorbov V. M. ${ }^{1}$, Movchan S. N. ${ }^{2}$, Solomonyuk D. N. ${ }^{1,2}$ \\ ${ }^{1}$ National University of Shipbuilding adm. Makarov, ${ }^{2}$ State Enterprise "Gas Turbine Scientific Production Complex "Zorya"-"Mashproekt" Mykolaiv, Ukraine
}

\begin{abstract}
The aim of this work is to determine the effect of the elements, which do not participate in heat transfer, on the mass of the regenerator of a gas turbine plant $\mathrm{X}$, as well as to define the restrictions that are imposed on the regenerator design based on the conditions of manufacturability, placement at the facility and transportability. This goal is achieved using an algorithm for finding rational geometric parameters of the heat exchange matrix with minimization of the regenerator mass by Newton's method. It has been determined that the mass of the heat exchange matrix can be $0.48-0.58$ of the mass of the regenerator. This makes it necessary, even at the initial design stages, to take into account the effect of the above factors on the mass of the regenerator and the choice of the rational geometrical parameters. A significant result of the studies performed is determination of the effect of dimensional restrictions and requirements for the shape of the regenerator to be increased in its mass. The values of the geometrical parameters of the heat exchange matrix were obtained, at which the mass of the regenerator takes on a minimum value. The significance of the work is that the obtained relationships between the mass of the regenerator and its geometry makes it possible to reduce the metal consumption of the regenerator and the gas turbine plant, which allows designing the heat exchangers for power plants.
\end{abstract}

Keywords: gas turbine plant, regenerator, heat-exchange matrix, heat effectiveness, pressure drops, mass, overall dimensions.

DOI: https://doi.org/10.52254/1857-0070.2021.2-50.04

UDC: 621.438

Regeneratoare de serpentină cu tuburi netede pentru centrale cu turbine cu gaz, optimizate în conformitate cu criteriul de greutate

Gorbov V.M., ${ }^{1}$ Movcean S.N., ${ }^{2}$ Solomoniuc D. N. ${ }^{2}$

${ }^{1}$ Universitatea Națională de Construcții Navale. adm. Makarov, ${ }^{2}$ Intreprindere de Stat "Complex ȘtiiințificoIndustrial de construire de turbine cu gaz "Zorea"-"Maşproiect", Nikolaev, Ucraina

Rezumat. Scopul lucrării este de a determina efectul asupra masei regeneratorului unei centrale cu turbină cu gaz a elementelor care nu participă la procesul de transfer de căldură, precum și restricțiile impuse la proiectarea regeneratorului în baza condițiilore de fabricare, punerea în funcție și transportabilitate. Acest obiectiv este realizat prin utilizarea unui algoritm pentru găsirea parametrilor geometrici raționali ai matricei de schimb de căldură cu minimizarea masei regeneratoare prin metoda Newton. Pentru a implementa acest algoritm, autorii au obținut relații pentru calcularea caracteristicilor de masă și dimensiune a matricei schimbului de căldură, a elementelor corpului și a regeneratorului în ansamblu. S-a stabilit, că masa matricei de schimb de căldură poate constitui 0,48-0,58 din masa regeneratorului, ceea ce duce la necesitatea de a lua în considerare influența factorilor menționați de mai sus asupra masei regeneratorului și alegerea parametrilor geometrici raționali deja la etapele inițiale de proiectare. Este prezentată o soluție tehnică, care a făcut posibilă reducerea masei colectoarelor pentru alimentarea și îndepărtarea lichidului de răcire din matricea de schimb de căldură de 3,2 ori și greutatea întregului schimbător de căldură cu $22 \%$. Semnificația lucrării constă în faptul, că relațiile obținute între masa regeneratorului și geometria acestuia fac posibilă reducerea consumului de metal al regeneratorului și al centralei cu turbină cu gaz și pot fi utilizate la proiectarea schimbătorilor de căldură pentru instalațiile energetice de putere.

Cuvinte-cheie: unitate de turbină cu gaz, regenerator, matrice de schimb de căldură, eficiență termică, pierdere de presiune, greutate, dimensiuni.

Гладкотрубные змеевиковые регенераторы газотурбинных установок, оптимизированные по критерию минимальной массы

Горбов В.М., ${ }^{1}$ Мовчан С. Н. ${ }^{2}$, Соломонюк Д. Н. ${ }^{2}$

${ }^{1}$ Национальный университет кораблестроения им. адм. Макарова, ${ }^{2}$ Государственное предприятие "Научно-производственный комплекс газотурбостроения "Зоря"-"Машпроект" Николаев, Украина

Аннотация Целью работы является определение влияния на массу регенератора газотурбинной уста- 
новки элементов, не принимающих участия в процессе передачи тепла, а также ограничений, которые накладываются на конструкцию регенератора исходя из условий технологичности, размещения на объекте и транспортабельности. Поставленная цель достигается за счет использования алгоритма поиска рациональных геометрических параметров теплообменной матрицы с минимизацией массы регенератора методом Ньютона. Для реализации данного алгоритма авторами получены соотношения для расчета массогабаритных характеристик теплообменной матрицы, корпусных элементов и регенератора в целом. Определено, что масса теплообменной матрицы может составлять 0,48-0,58 от массы регенератора, что приводит к необходимости уже на начальных этапах проектирования учитывать влияние вышеуказанных факторов на массу регенератора и выбор рациональных геометрических параметров. Существенным результатом проведенных исследований является определение влияния габаритных ограничений и требований к форме регенератора на увеличение его массы (до 8\% и 50\% соответственно). Получены значения геометрических параметров теплообменной матрицы, при которых масса регенератора принимает минимальное значение, а именно диаметры труб 20-22 мм, количестве ходов теплоносителя 4-8 (при тепловой эффективности регенератора 0,8-0,85) и 6-10 (при 0,9 и выше), относительный поперечный шаг труб в пучке 1,25-1,38. Приведено техническое решение, которое позволило уменьшить массу коллекторов для подвода и отвода теплоносителя из теплообменной матрицы в 3,2 раза, а массу всего теплообменника - на $22 \%$. Значимость работы состоит в том, что полученные зависимости между массой регенератора и его геометрией позволяют снизить металлоемкость регенератора и газотурбинной установки и могут быть использованы при проектировании теплообменников для силовых энергетических установок различных типов.

Ключевые слова: газотурбинная установка, регенератор, теплообменная матрица, тепловая эффективность, потери давления, масса, габариты.

\section{ВВЕДЕНИЕ}

Благодаря высокой удельной мощности, малым массогабаритным показателям, высокой маневренности и простоте в обслуживании и ремонте газотурбинные установки (ГТУ) получили широкое распространение в энергетике, транспортировании газа, судо- и кораблестроении, на транспорте [1-3].

Существенным недостатком ГТУ простого цикла является относительно низкие значения их коэффициента полезного действия (КПД).

На сегодня КПД энергетических и транспортных ГТУ мощностью от 15 до нескольких сотен мегаватт составляет 35-38\%. У ГТУ мощностью 5-10 МВт КПД находится на уровне $30-32 \%$, у ГТУ малой мощности (2,5-3 МВТ) - около 28-30\%, у микро-ГТУ еще ниже [1-3]. До середины 90-х годов XX века КПД ГТУ в основном увеличивался за счет повышения начальной температуры газа $\left(t_{3}\right)$. На сегодня практически достигнут уровень $t_{3}=1300-1400{ }^{\circ} \mathrm{C}$, но дальнейший рост температур без разработки новых систем охлаждения и внедрения новых жаростойких материалов невозможен [1-4].

В связи с этим, в последнее время интерес стали вызывать ГТУ сложных циклов (в том числе и с регенерацией теплоты), в составе которых применяются различные теплообменные аппараты [5-12].

В конце XX века фирмами Rolls-Royce и Westinghouse для военно-морских сил США была разработана газотурбинная установка
$W R-21$ с промежуточным охлаждением и регенерацией (мощность 25,2 МВт, КПД $43 \%$ ) [8]. Для привода судового генератора фирмой Solar Turbines Inc. предложена газотурбинная установка с регенерацией Mercury-50 (мощность 4,6 МВт, КПД 38,5 \%) [9]. Регенеративные ГТУ для судовых и стационарных установок также разрабатывались Государственным предприятием «Научно-производственный комплекс газотурбостроения (ГП НПКГ) «Зоря»-«Машпроект» [3, 11].

Анализ перспектив применения регенеративных ГТУ в газотранспортной системе Украины проведен в [11]. Рядом фирм предложены новые регенераторы для модернизации регенеративных газоперекачивающих агрегатов [12].

Оптимизационные расчеты схем ГТУ с простой регенерацией $[13,14]$ и конструктивные проработки [8-9], показали, что регенерация теплоты в среднем повышает КПД на 6-8 \% (абс.) на номинальном режиме и обеспечивает его высокие значения на частичных режимах. В [3, 11] отмечено, что высокий уровень КПД достигается уже при $t_{3}=900$ $950{ }^{\circ} \mathrm{C}$, что позволяет обойтись без охлаждения турбинных ступеней и использовать относительно недорогие жаростойкие материалы и проверенные временем технологии. В работах [10, 15-17] показано, что особенно перспективной регенерация является для микротурбинных двигателей с мощностью в несколько сот киловатт. 
Кроме исследований схем ГТУ с простой регенерацией продолжаются работы по созданию регенеративных газотурбинных установок сложных циклов. Так Alklaibi и др. [18] рассмотрели ГТУ с воздушной утилизационной турбиной. Ожидается, что такая схема увеличит мощность ГТУ на 6-16\%, а общий КПД установки повысит до $45 \%$.

В [19] выполнен термодинамический анализ ГТУ с регенерацией и промежуточным охлаждением воздуха, показано, что их КПД на $2-5 \%$ (абс.) больше, чем у регенеративных. В [20] рассмотрена схема ГТУ с насыщением воздуха парами воды и регенерацией, КПД которой достиг $61,4 \%$.

В [21] рассмотрена полузамкнутая ГТУ, в которой через регенератор, расположенный на линии продуктов сгорания высокого давления, проходит только часть газового потока. В [22] рассмотрена схема ГТУ с промежуточной регенерацией, в которой регенератор размещен между турбинами. Показано, что в данных схемах можно получить больший КПД, чем в обычном регенеративном цикле.

Существенным недостатком работ [13-22] можно считать то, что при анализе схем ГТУ и выборе их оптимальных параметров авторы ограничиваются только термодинамическим анализом и не учитывают влияние параметров цикла на массу и габариты регенератора.

В $[5,8-19,11]$ также указано, что основную сложность при разработке регенеративных ГТУ представляет создание высокоэффективного и надежного регенератора, который имел бы приемлемые массогабаритные и стоимостные показатели.

В качестве регенераторов в ГТУ используются рекуперативные теплообменники с трубной и пластинчатой поверхностями теплообмена $[6-12,16,17]$. Регенераторы с трубными поверхностями имеют большие массу и габариты, чем пластинчатые, однако они менее чувствительные к термическим нагрузкам, которые возникают в процессах пуска, остановки и переходных режимов работы ГТД, и способны обеспечить надежную работу при высоких температурах и давлениях рабочих сред, поэтому считаются более долговечными и надежными в эксплуатации.

В связи с большой массой и габаритами регенератора актуальной задачей при проектировании ГТУ сложных циклов есть разработка такой конструкции теплообменника, которая бы обеспечила заданные параметры ГТУ при минимальных массах и габаритах, что особенно актуально для транспортных и микротурбинных установок [10, 15-17].

В $[5,10,15-17,22-25]$ отмечается, что проектирование регенератора является многоцелевым оптимизационным процессом по поиску компромиссного решения между высокой тепловой эффективностью, низкими потерями давления, малыми массогабаритными показателями и низкой стоимостью.

Данную проблему можно решить за счет выбора рациональных значений геометрических параметров теплообменной матрицы, что является сложной задачей, учитывая большое количество существующих поверхностей теплообмена и их геометрических параметров, и требует совершенствования методов расчетов теплообменного оборудования $[7,10,16,17]$.

В литературе, посвященной проектированию теплообменных аппаратов, приведены общие рекомендации по уменьшению массы и габаритов теплообменников: уменьшение размеров каналов, увеличение скоростей теплоносителей, использование интенсификаторов теплоотдачи [23, 25]. Характеристика методов оптимизации конструкции регенераторов с общей блок-схемой выполнения оптимизационных расчетов приведена в [17].

В [24] разработано программное обеспечение по оптимизации конструкции регенераторов ГТУ и расчетам экономического эффекта. В зависимости от приоритетов проектирования целевой функцией может быть компактность матрицы, потери давления или стоимость изделия.

В [26] разработана методика выбора оптимальных пар существующих пластинчаторебристых поверхностей теплообмена, которая обеспечивает минимальный объем теплообменной матрицы, но не был выполнен анализ влияния их геометрических параметров на массогабаритные показатели регенератора.

В [28] использован метод минимизации генерации энтропии для анализа пластинчатых теплообменников с различной конфигурацией поверхности теплообмена. В [29] авторы нашли оптимальные параметры волнообразных каналов с максимизацией теплопередачи и минимизацией потерь давления. В [30] выполнена многопараметрическая оптимизация конструкции регенератора исходя из теории рассеяния энтропии.

В большинстве современных методик поиска оптимальных геометрических параметров регенератора рассматриваются только 
характеристики его теплообменной матрицы. При этом в любом трубчатом теплообменном аппарате можно выделить следующие конструктивные элементы:

- теплообменная матрица, в которой осуществляется процесс передачи тепла;

- элементы, которые не принимают участия в процессе теплопередачи, а именно:

- калачи, переходы, по которым теплоносители переходят из одного участка матрицы в другой;

- коллекторы, по которым теплоноситель распределяется по участкам теплообменной матрицы и отводится из нее;

- отводы, при помощи которых теплообменная матрица присоединяется к коллекторам;

- перегородки, которые организуют движение теплоносителя внутри матрицы, придают ей жесткость и служат опорами для труб;

- корпус с опорами и изоляцией.

Поскольку в $[12,23]$ показано, что масса матрицы может составлять $0,5-0,7$ от массы всего регенератора, важной задачей на начальных этапах проектирования становится не только получение теплообменной матрицы с минимальной массой и габаритами, но и минимизация массы остальных элементов регенератора. Опыт проектирования регенеративных ГТУ показывает $[8,11,12]$, что при проектировании регенератора следует учитывать массовые и габаритные ограничения, которые налагаются на конструкцию регенератора из условий технологичности, размещения на объекте и транспортирования. Особенно это актуально для транспортных установок и микро-ГТУ $[10,15-17,31]$. Подобные вопросы возникают и при модернизации существующих регенеративных установок путем замены вышедших из строя регенераторов новыми [12]. В этом случае масса и габариты нового регенератора должны соответствовать параметрам старого, чтобы установить теплообменник на существующем фундаменте и скомпоновать его с имеющимся оборудованием при минимальных переделках установки. Влияние указанных ограничений на массовые показатели регенератора еще недостаточно изучено. Например, в [27] при анализе цилиндрического регенератора были введены ограничения на шаги труб, длину труб и размеры трубного пучка, но не было проведено исследование их влияния на массу регенератора.
В $[23,31]$ проанализировано влияние геометрических параметров теплообменной матрицы на ее объем и фронтальные площади, но не было определено, как изменится масса регенератора при перекомпоновке матрицы в случае, когда эти параметры выходят за установленные границы.

Таким образом, возникла задача определения влияния геометрических параметров теплообменной матрицы регенератора на его массу, с учетом элементов, не принимающих участия в процессе передачи тела, а также ограничений, которые накладываются на конструкцию исходя из условий технологичности, размещения на объекте и транспортирования.

\section{МЕТОДЫ}

В качестве модели для анализа выбран гладкотрубный змеевиковый регенератор установки ГТУ-16Р (рис. 1), разработанной ГП НПКГ "Зоря"-"Машпроект" для привода нагнетателя природного газа [11]. Основные расчетные параметры ГТУ при стандартных атмосферных условиях в условиях компрессорной станции приведены в таблице 1.

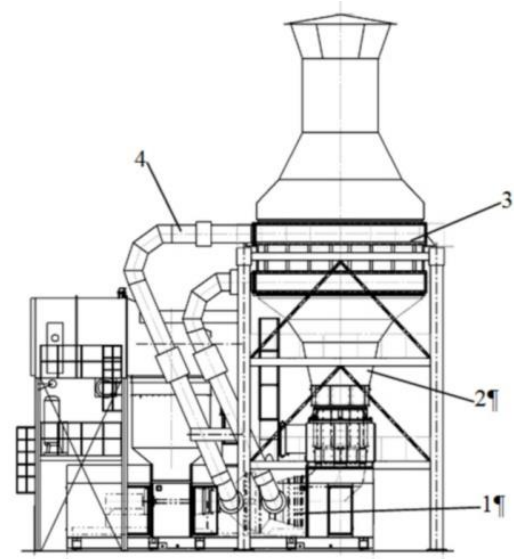

1 - контейнер ГТУ; 2 - газоход; 3 -регенератор; 4 - воздуховоды.

Рис. 1 Компоновка ГТУ мощностью 16 МВт с регенерацией теплоты ${ }^{1}$

Таблица $1^{2}$.

Основные проектные параметры ГТУ мощностью 16 МВт для привода нагнетателя природного газа 3.

\begin{tabular}{|l|c|}
\hline \multicolumn{1}{|c|}{\begin{tabular}{l}
\multicolumn{1}{|c|}{ Параметр } \\
(Parameter)
\end{tabular}} & $\begin{array}{c}\text { Значение } \\
\text { (Value) }\end{array}$ \\
\hline $\begin{array}{l}\text { Мощность на выходном валу, } \\
\text { МВт } \\
\text { (Power on output shaft, MW) }\end{array}$ & 16 \\
\hline КПД ГТД, \% & 40,3 \\
\hline
\end{tabular}




\begin{tabular}{|c|c|}
\hline $\begin{array}{l}\text { (Gas turbine engine thermal effi- } \\
\text { ciency, \%) }\end{array}$ & \\
\hline $\begin{array}{l}\text { Степень повышения давления } \\
\text { (Overall pressure ratio) }\end{array}$ & 5,75 \\
\hline $\begin{array}{l}\text { Температура газа на входе в } \\
\text { турбину, }{ }^{\circ} \mathrm{C} \\
\text { (Turbine gas inlet temperature, }{ }^{\circ} \mathrm{C} \text { ) }\end{array}$ & 980 \\
\hline $\begin{array}{l}\text { Расход воздуха на входе в ГТД, } \\
\text { кг/c } \\
\text { (Gas turbine inlet gas mass flow, } \\
\mathrm{kg} / \mathrm{sec} \text { ) }\end{array}$ & 74,9 \\
\hline $\begin{array}{l}\text { Степень регенерации теплоты } \\
\text { (Regenerator effectiveness) }\end{array}$ & 0,85 \\
\hline $\begin{array}{l}\text { Температура воздуха на входе в } \\
\text { регенератор, }{ }^{\circ} \mathrm{C} \\
\text { (Regenerator air inlet temperature, } \\
{ }^{\circ} \mathrm{C} \text { ) }\end{array}$ & 223 \\
\hline $\begin{array}{l}\text { Температура газа на входе в } \\
\text { регенератор, }{ }^{\circ} \mathrm{C} \\
\text { (Regenerator gas inlet tempera- } \\
\text { ture, }{ }^{\circ} \mathrm{C} \text { ) }\end{array}$ & 590 \\
\hline $\begin{array}{l}\text { Относительные потери давления } \\
\text { (суммарные), \% } \\
\text { (Relative pressure drops (overall), } \\
\% \text { ) }\end{array}$ & 4 \\
\hline
\end{tabular}

Регенератор (рис. 2) состоит из двух секций, которые при монтаже устанавливаются параллельно. Схема движения теплоносителей - многократный перекрестный ток с общим противотоком. Поверхность теплообмена набрана из плоских трубных пучков (змеевиков), размещенных в прямоугольном коробе, который является частью газохода ГТД. Теплообменные трубы размещены в шахматном порядке. Выхлопные газы обтекают трубы снаружи, воздух осуществляет несколько ходов в середине труб и подводится (отводится) по круглым коллекторам. Трубы соединяются с коллекторами с помощью гнутых отводов. Переход воздуха из одного хода в другой выполнен по калачам. Между ходами по периметру газохода установлены вытеснители.

Особенности конструкции регенератора задекларированы в патенте на полезную модель UA 78601 U [34].

В основу алгоритма расчета регенератора был положен метод $\varepsilon-\mathrm{NTU}$ [32]. В соответствии ним основной характеристикой регенератора, как и любого теплообменника, есть

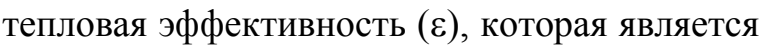
отношением теплового потока в регенераторе $\left(Q_{r e g}\right)$ к максимальному тепловому потоку, который можно передать от горячего теплоносителя к холодному $\left(Q_{\max }\right)$.

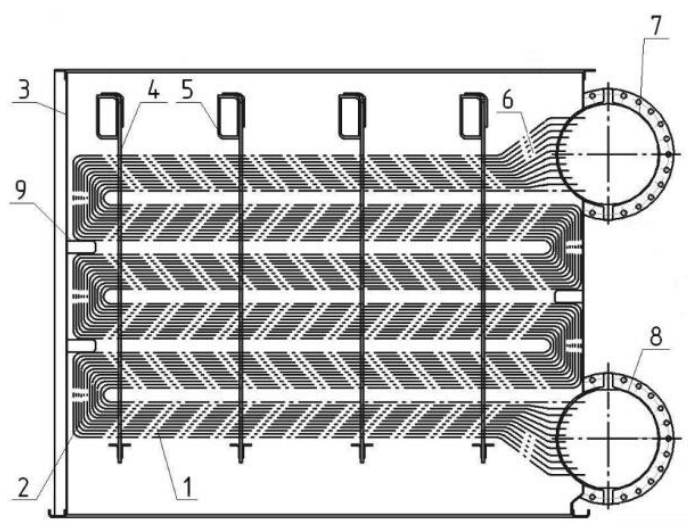

1 - теплообменный пакет из плоских змеевиков; 2 - калачи; 3 - корпус; 4 - проставки; 5 -балки; 6 -гнутые отводы; 7 - раздаточный воздушный коллектор; 8 - сборной воздушный коллектор; 9 вытеснитель.

Рис. 2. Гладкотрубный змеевиковый регенераTop. ${ }^{4}$

Для расчета тепловой эффективности используются зависимости вида [32, 33]:

$$
\varepsilon=Q_{\text {reg }} / Q_{\max }=f\left(\mathrm{NTU}, W_{\text {rel }}\right),
$$

где $W_{\text {rel }}=W_{\min } / W_{\max }, \mathrm{NTU}=k F / W_{\min }$ - количество единиц переноса теплоты; $W_{\min }$ и $W_{\max }$, $\mathrm{BT} / К$ - минимальный и максимальный водяные эквиваленты теплоносителей $\left(W_{\text {hot }}=G_{\text {hot }} \cdot c_{p \text { hot }}, W_{\text {cold }}=G_{\text {cold }} \cdot c_{p c o l d}\right), G, \kappa г / \mathrm{c}-$ расход теплоносителя, $F$, м $^{2}$ - площадь поверхности теплообмена со стороны соответствующего теплоносителя (горячего или холодного); $k, \mathrm{BT} /\left(\mathrm{m}^{2} \mathrm{~K}\right)$ - коэффициент теплопередачи от горячего теплоносителя к холодному, отнесенный к площади поверхности теплообмена со стороны соответствующего теплоносителя; $c_{p}$, Дж/(кг К) - изобарная теплоемкость теплоносителя.

В качестве гидравлической характеристики регенератора используется величина суммарных относительных потерь давления:

$$
\delta P_{\Sigma}=\delta P_{\text {hot }}+\delta P_{\text {cold }},
$$

где $\delta P_{\text {hot }}=\Delta P_{\text {hot }} / P_{\text {hot in }}$ и $\delta P_{\text {cold }}=\Delta P_{\text {cold }} / P_{\text {cold in }},-$ относительные потери давления горячего и холодного теплоносителей в регенераторе, $\Delta P_{\text {hot }}$ и $\Delta P_{\text {cold }}$ - абсолютные потери давления горячего и холодного теплоносителей в регенераторе; $P_{\text {hot in }}, P_{\text {cold in }}$, Па - давление холод- 
ного и горячего теплоносителей на входе в регенератор.

Из зависимостей (1), (2) авторами была получена система уравнений, которая связывает относительную массу регенератора $\left(m_{q}\right)$ с тепловой эффективностью, относительными потерями давления теплоносителей, параметрами теплоносителей и геометрическими параметрами теплообменной матрицы:

$$
m_{q}=f\left[\begin{array}{l}
\varepsilon, k_{F}, k_{M}, k_{L}, \rho_{M}, \\
\left(\delta P, P_{i n}, T_{i n}, l, \psi, s_{q}\right)_{h o t}, \\
\left(\delta P, P_{i n}, T_{i n}, l, \psi, s_{q}\right)_{c o l d}, \\
W_{\min }, W_{r e l}
\end{array}\right],
$$

где $m_{q}=M_{\text {reg }} / W_{\text {min }}, \kappa г /(\mathrm{Bт} / \mathrm{K})$ - масса регенератора, отнесенная к минимальному водяному эквиваленту, $\rho_{M}=\rho_{s t} \cdot V_{s t} / V_{M}, \kappa г / \mathbf{m}^{3}-$ условная плотность теплообменной матрицы, зависит от геометрии каналов и плотности материала матрицы, $V_{M}$, м $^{3}$ - объем матрицы, $V_{s t}, \mathrm{M}^{3}$ - объем матрицы за исключением объема каналов для прохода теплоносителей,

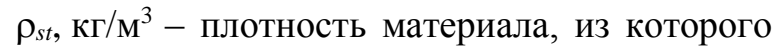
выполнена теплообменная матрица, $k_{F}-$ коэффициент запаса поверхности; $k_{M}=M_{M} / M_{r e g}-$ отношение массы матрицы к массе всего теплообменника, $k_{L}=L / L_{x}-$ отношение длины хода теплоносителя в активной части матрицы $(L)$ к общей длине его хода в теплообменной матрице $\left(L_{x}\right)$; $s_{q}=S / W, \mathrm{M}^{2} /(\mathrm{BT} / \mathrm{K})$ - отношение площади для прохода теплоносителя $(S)$ к величине водяного эквивалента теплоносителя; $\psi=F / V_{M}, \mathrm{M}^{2} / \mathrm{M}^{3}$ - коэффициент компактности поверхности теплообмена со стороны соответствующего теплоносителя.

Авторами разработан алгоритм определения рациональных параметров регенератора, в котором минимизация массы выполнена методом поиска экстремума функции многих переменных, особенностью которого является квадратичная аппроксимация целевой функции с использованием матрицы ее вторых производных (метод Ньютона) [35]. С помощью этого алгоритма возможно подобрать такие значения геометрических пара- метров теплообменной матрицы, которые обеспечивают минимальную массу регенератора при заданных значениях тепловой эффективности регенератора и относительных потерь давления теплоносителей.

Уравнение (3) представляет относительную массу регенератора $m_{q}$ в виде функциональной зависимости от вектора, в котором собраны значения степени регенерации, потерь давления и параметров теплоносителей, а также геометрии теплообменной матрицы:

$$
m_{q}=F(\bar{x})=F\left(x_{1}, x_{2}, \ldots, x_{n}\right)
$$

Необходимым условием наличия экстремума функции нескольких переменных $F(x)$ в точке $x^{*}$ есть равенство нулю ее градиента в этой точке:

$$
F^{\prime}\left(x^{*}\right)=0 \text {. }
$$

Значение вектора $\overline{x^{*}}=\left(x^{*}, x_{1}{ }_{2}, \ldots, x^{*}{ }_{n}\right)$, которое обеспечивает минимум функции $F(x)$ с заданной точностью $\delta$, будет решением поставленной задачи.

Зависимость (3) включает к себе величины $k_{M}$ и $k_{L}$, которые характеризуют элементы регенератора, не принимающие участия в процессе передачи тепла (корпус, изоляция, коллекторы, калачи, отводы), что в отличие от других аналогичных зависимостей дает возможность проанализировать их влияние на массогабаритные показатели регенератора.

Исходными данными для расчета являются параметры теплоносителей на входе в регенератор (табл. 1), тип и геометрические параметры теплообменной матрицы (рис. 3):

- внешний $\left(d_{\text {out }}\right)$ и внутренний $\left(d_{\text {inn }}\right)$ диаметры труб;

- толщина стенки труб $\left(\delta_{t}\right)$;

- продольный $\left(s_{1}\right)$ и поперечный $\left(s_{2}\right)$ шаги труб в пучке;

- количество ходов теплоносителя $\left(n_{x}\right)$.

Заданные значения тепловой эффективности регенератора и потерь давления в регенераторе обеспечиваются выбором длины труб $(L)$; количества труб в ряду поперек потока 

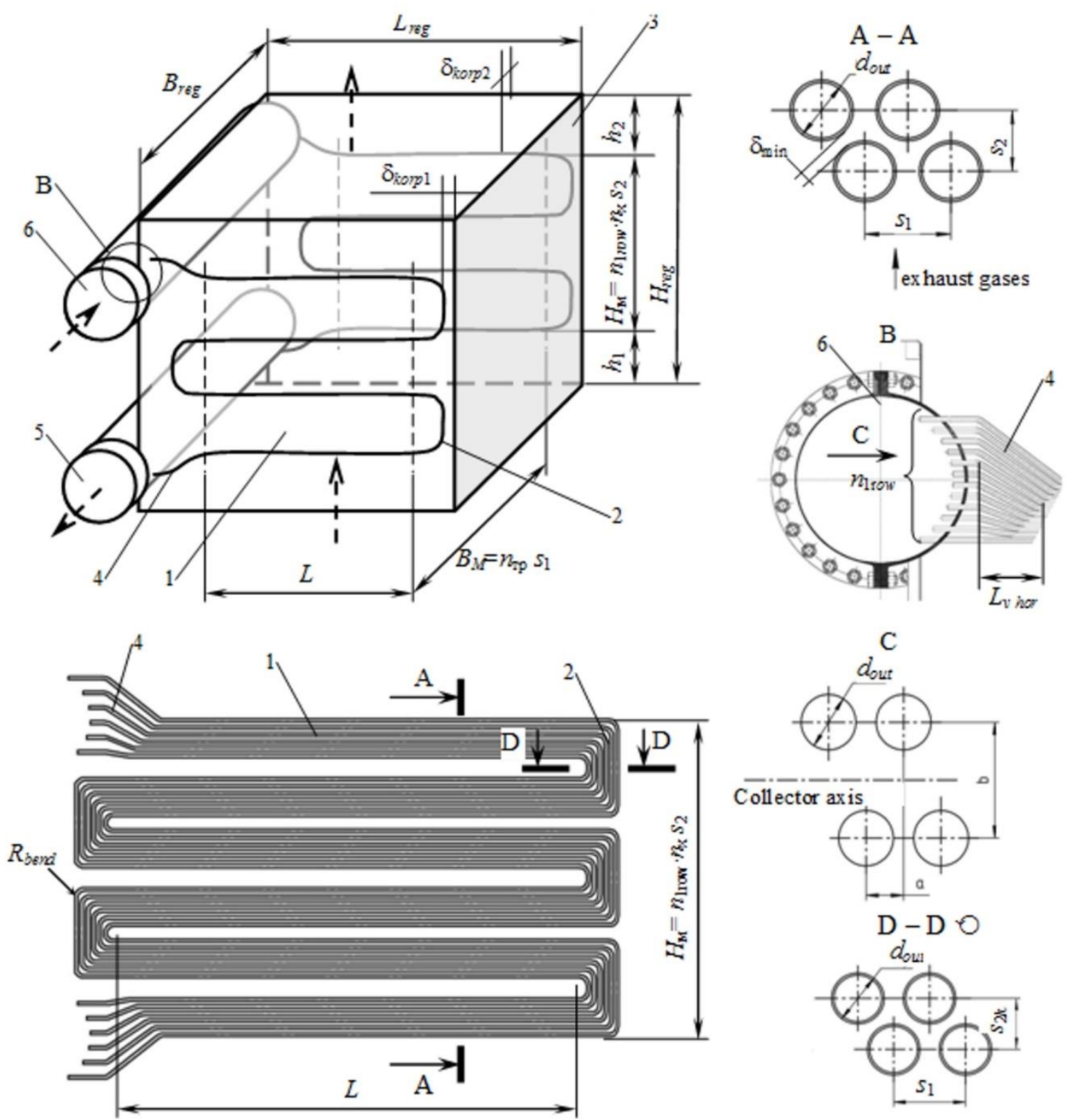

1 - теплообменный пакет из плоских змеевиков; 2 - калачи; 3 -корпус; 4 - гнутые отводы; 5 - сборный воздушный коллектор; 6-раздаточный воздушный коллектор;

Рис. 3. Геометрические характеристики гладкотрубного змеевикового регенератора ГТУ. ${ }^{5}$

$\left(n_{\text {tube }}\right)$ и количеством рядов труб в одном ходу (n $\left.n_{\text {row }}\right)$.

В процессе расчета определяются следующие геометрические параметры регенератоpa:

- количество рядов труб в теплообменнике

$$
n_{\text {row }}=n_{1 \text { row }} \cdot n_{x}
$$

- высота матрицы, м

$$
H_{M}=n_{\text {row }} \cdot s_{2}
$$

- ширина матрицы, м

$$
H_{M}=n_{\text {row }} \cdot s_{2}
$$

- объём матрицы, м ${ }^{3}$

$$
V_{M}=L \cdot H_{M} \cdot B_{M}
$$

- удельный объём матрицы, м³/(Вт/К)

$$
v_{q}=V_{M} / W_{\min }
$$

- площадь для прохода теплоносителя в середине труб, м $^{2}$

$$
S_{i n n}=\pi \cdot d_{i n n}^{2} / 4 ;
$$


- площадь для прохода теплоносителя между трубами, м²

$$
S_{\text {out }}=L \cdot n_{\text {tube }} \cdot\left(s_{1}-d_{\text {out }}\right) \text {; }
$$

- шаг между проставками, м $s_{p l}$;

- количество проставок

$$
n_{p l}=B_{M} / s_{p l}
$$

- поперечный шаг между трубами в калачах, м $s_{2 k}$;

- вертикальный шаг труб в отводах в месте входа в коллектор, м $b$;

- средняя длина отвода по горизонтали, м $L_{v}$ hor

- средняя длина пути теплоносителя в отводах

$L_{v}=L_{v \text { hor }} / \cos \left(\operatorname{arctg}\left(n_{1 \text { row }} \cdot\left(b-\mathrm{s}_{2}\right) / L_{\mathrm{v} \text { hor }}\right)\right)$;

- длина пути теплоносителя в трубах, который приходится на один ход теплоносителя, м

$$
L_{x}=L+\mathrm{n}_{p l} \cdot \mathrm{d}_{p l}+s_{2 k} \cdot n_{\text {lrow }}+H_{M} / n_{x}+L_{\mathrm{v}} / n_{x},
$$

- ориентировочная длина корпуса регенератора, м,

$$
L_{r e g}=L_{M}+L_{v \text { hor }}+2 d_{k o r p 1} \text {, }
$$

где $\delta_{k o r p 1}$, м - конструктивный зазор между калачами и корпусом;

- ориентировочная высота регенератора, м

$$
H_{r e g}=H_{M}+h_{1}+h_{2}
$$

где $h_{1}, h_{2}$, м - конструктивные добавки на высоту матрицы для размещения элементов корпуса;

- ориентировочная ширина корпуса теплообменника, м

$$
B_{\text {reg }}=B_{M}+2 d_{k o r p 2},
$$

где $\delta_{\text {korp } 1,}$ м - конструктивный зазор между трубами и корпусом;

- площадь поверхности теплообмена (по внутренней стороне), м $^{2}$

$$
F=\pi \cdot n_{\text {row }} \cdot n_{\text {row }} \cdot d_{\text {inn }} \cdot L \text {; }
$$

- удельная площадь теплообменной поверхности, м²/(Вт/K)

$$
f_{q}=F / W_{\min }
$$

- объём матрицы, м ${ }^{3}$

$$
V_{M}=n_{\text {row }} \cdot n_{1 \text { row }} \cdot L \cdot s_{1} \cdot s_{2}
$$

- масса активной части матрицы, кг

$$
M_{M}=V_{M} \cdot \rho_{M}
$$

- удельная масса матрицы (отнесенная к минимальному водяному эквиваленту), кг/(Вт/К)

$$
m_{q M}=M_{M} / W_{\min }
$$

- масса всей матрицы (с учетом участков труб, которые не принимают участие в теплообмене), кг

$$
M_{M \Sigma}=M_{M} / k_{L}
$$

- масса корпуса

$$
M_{\text {korp }}=2 H_{r e g} \cdot\left(B_{r e g}+L_{r e g}\right) \cdot \rho_{k o r p} ;
$$

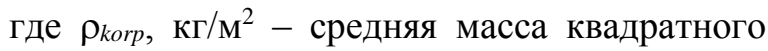
метра поверхности корпуса с изоляцией, опорами, фланцами и балками;

- масса проставок

$$
M_{p l}=n_{p l} \cdot d_{p l} \cdot H_{r e g} \cdot B_{M} \cdot \rho_{s t}
$$

- масса коллекторов

$$
M_{\kappa}=H_{r e g} \cdot \rho_{s t} \cdot \pi\left(D_{\text {out }}{ }^{2}-D_{\text {inn }}{ }^{2}\right) \text {, }
$$

где $D_{\text {out }}, D_{i n n}$, м - внешний и внутренний диаметры коллекторов;

- масса всего теплообменника, кг,

$$
M_{r e g}=M_{M \Sigma}+M_{k o r p}+M_{p l}+M_{\kappa} .
$$

На основании полученных данных рассчитываются величины $m_{q}, k_{M}$. 


\section{РЕЗУЛЬТАТЫ}

Уменьшение диаметра труб при неизменных других параметрах теплообменной матрицы приводит к уменьшению ее площади, объема и массы (рис. 4, а), что совпадает с выводами [23, 31-33]. При этом возрастает количество труб в пакете, что повышает тру-

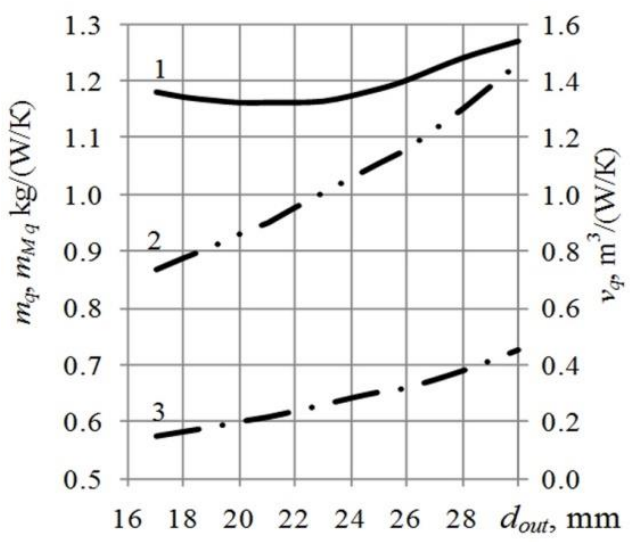

a) доемкость изготовления теплообменника. Масса же всего регенератора вначале уменьшается, а при малых диаметрах труб начинает возрастать вследствие уменьшения величины $k_{M}$ (рис. 4, б), и при $d_{\text {ou }}=20-22$ мм образуется минимум. В общем, масса теплообменной матрицы составляет $0,48-0,58$ от массы регенератора (рис. 4, б).

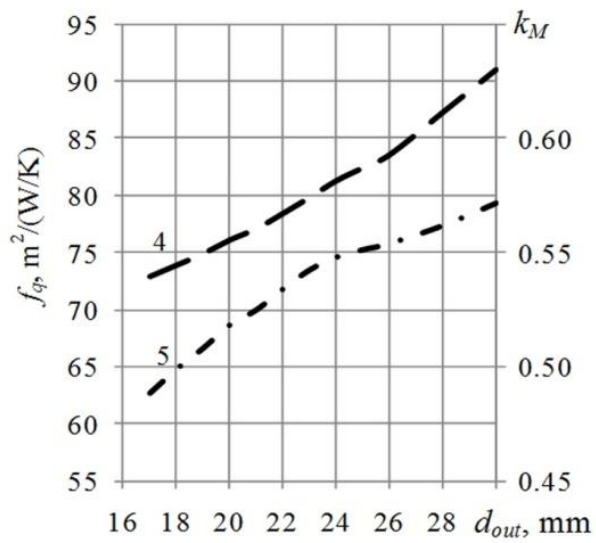

б)

1 - относительная масса регенератора; 2 - относительный объём теплообменной матриць; 3 относительная масса теплообменной матрищы; 4 - относительная площадь поверхности теплообмена; 5 - отношение массы теплообменной матриџы к массе регенератора

Рис. 4. Зависимость массогабаритных характеристик гладкотрубного змеевикового регенератора от диаметра труб (другие геометрические параметры теплообменной матрицы постоянные). ${ }^{6}$

Расчеты показали, что изменение толщины стенок труб в диапазоне от 1 мм до 2 мм не оказывает существенного влияния на процесс теплопередачи и величины потерь давления. Площадь поверхности теплообмена при этом остается неизменной, а относительная масса теплообменной матрицы увеличивается с 0,723 кг/(Вт/К) до 1,48 кг/(Вт/К). Соответственно, относительная масса регенератора увеличивается с 1,35 кг/(Вт/К) до 2,49 кг/(Вт/К). С учетом этого рекомендуется ограничивать минимальное значение толщины стенок труб с учетом требований технологичности или надежности. Технологически минимальные допустимые значения диаметра труб и толщины стенки ( $d_{\text {out } \min }$ и $\left.\delta_{t \min }\right)$ определяются существующими типоразмерами, возможностью выполнения качественного гиба и сварки труб и из условия прочности и заданного ресурса [36]. Для гладких труб при pecypce 100 тыс. часов минимальную толщину можно принять 1 мм.

Уменьшение диаметра трубок приводит к уменьшению ширины регенератора и увеличение его длины и высоты (рис. 5), но эти величины могут ограничиваться:

- размерами помещения, в котором размещается установка;
- транспортным габаритом $L_{r e g} \leq 3 \mathrm{~m}$, $B_{r e g} \leq 9 \mathrm{M}, H_{r e g} \leq 3 \mathrm{M}$;

- требованиями, которые накладываются заказчиком;

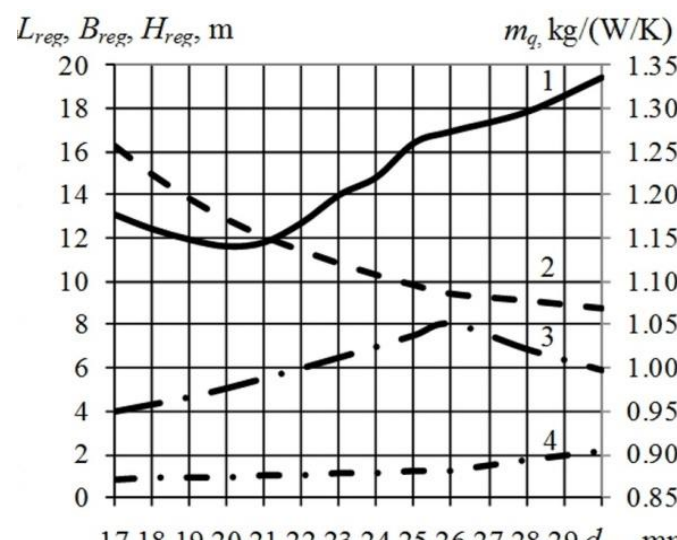

$17181920212223242526272829 d_{\text {out }}, \mathrm{mm}$

1 - относительная масса $\left(m_{q}\right)$; $2-$ длина $\left(L_{r e g}\right)$; 3 - ширина $\left(B_{\text {reg }}\right)$; 4 - высота $\left(H_{\text {reg }}\right)$.

Рис. 5. Влияние диаметра труб на массу и габариты гладкотрубного змеевикового регенераTopa $^{7}$

- габаритами заменяемого регенератора, его фундамента, габаритами существующего 
оборудования (при модернизации существующих установок).

Например, новые регенераторы для установки ГТК-10, которые предназначены для замены существующих пластинчатых регенераторов, исходя из условия размещения на газокомпрессорных станциях, должны отвечать габаритам $L_{\text {reg }} \leq 1,8$ м и $B_{\text {reg }} \leq 5$ м, чтобы соответствовать существующим газоходам и фундаментам [12].

Конструкции, которые не отвечали заданным габаритным ограничениям, были отброшены, и вместо них выбирались те, которые при выполнении поставленных ограничений имели наименьшую массу.

Вследствие этого (рис. 6) при некоторых диаметрах труб масса теплообменника увеличилась:

- на 6 \% при введении ограничения на ширину канала (при $d_{\text {out }}=25$ мм);

- на $8 \%$ при введении ограничения на длину канала (при $d_{\text {out }}=18$ мм).

Если рассматривать регенератор с учетом его компоновки с газотурбинным двигателем и размеров газоходов для подвода и отвода выхлопных газов, то более рациональной конструкцией теплообменника будет та, которая имеет поперечное сечение, близкое к квадрату (заданное соотношение сторон $\left.\left(L_{r e g} / B_{r e g}\right)_{g i v}=1\right)$. Когда одна из сторон теплообменника значительно больше другой, то угол раскрытия диффузора газохода по данной стороне будет наибольшим, что приведет к большим потерям давления в газоходе. Для их уменьшения придется увеличить длину диффузора, что приведет к увеличению массогабаритных показателей, или установить в

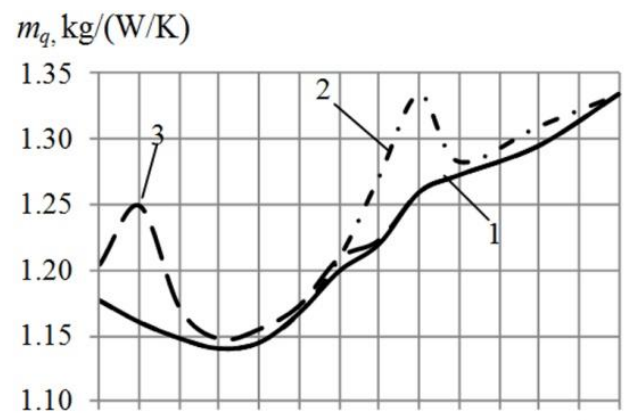

$171819202122232425262728 d_{\text {out }}, \mathrm{mm}$ газоходе направляющие устройства, что приведет к усложнению конструкции и трудностям в отладке установки.

При замене старых регенераторов (как это происходит с регенераторами ГТК-10) ограничение на соотношение сторон нового регенератора накладывается исходя из соотношения сторон старого теплообменника (для ГТК-10 $\left.\left(L_{\text {reg }} / B_{\text {reg }}\right)_{g i v}=2,8\right)$.

Соблюдение при проектировании точного заданного соотношения сторон $\left(L_{\text {reg }} / B_{\text {reg }}\right)=\left(L_{\text {reg }} / B_{\text {reg }}\right)_{g i v}$ может привести к росту массы регенератора до $50 \%$ (рис. 6).

Рекомендуется сделать это условие менее жестким. Так при допустимом отклонении $L_{\text {reg }} / B_{\text {reg }}$ от заданного значения на $30 \%$ (т.е. $\left.0.7\left(L_{\text {reg }} / B_{\text {reg }}\right)_{\text {giv }} \leq\left(L_{\text {reg }} / B_{\text {reg }}\right) \leq 1.3\left(L_{\text {reg }} / B_{\text {reg }}\right)_{\text {giv }}\right)$ увеличение массы не превышает $10 \%$ (рис. 6 , линия 5).

Также необходимо указать, что введение габаритных ограничений приводит к изменению значений диаметров труб, при которых получена минимальная масса (рис. 6). Так при отсутствии ограничений минимальная масса регенератора была получена при $d_{\text {out }}=20$ мм, при ограничении одного из габаритов $-d_{\text {out }}=21$ мм, при ограничении соотношения сторон $d_{\text {out }}=22 \mathrm{\text {м. }}$

Увеличение количества ходов теплоносителя приближает значение эффективности многоходового теплообменника к противо точному. Одновременно с этим возрастает сопротивление матрицы, для компенсации чего приходится увеличивать площадь для $m_{q}, \mathrm{~kg} /(\mathrm{W} / \mathrm{K})$

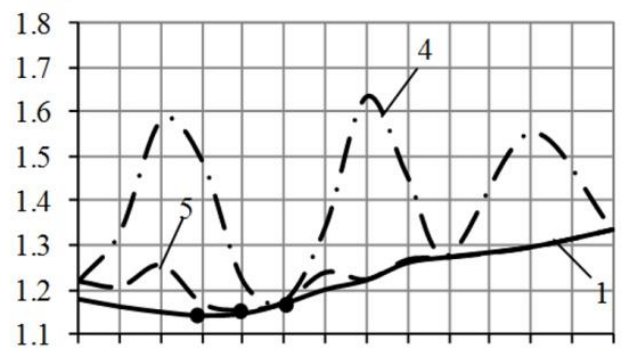

$17181920212223242526272829 d_{\text {out }}, \mathrm{mm}$

1 - расчет без ограничений габаритов; 2 - расчет при ограничении иирины $\left(B_{\text {reg }}\right) ; 3$ - расчет при ограничении длинь $\left(L_{r e g}\right) ; 4$ - расчет при жестком ограничении формы поперечного сечения регенераmopa $L_{r e g} / B_{\text {reg }}=\left(L_{r e g} / B_{r e g}\right)_{g i v} ; 5-$ pacyem npu $0.7\left(L_{r e g} / B_{r e g}\right)_{g i v} \leq L_{r e g} / B_{r e g} \leq 1.3\left(L_{r e g} / B_{r e g}\right)_{g i v}$

Рис. 6. Влияние диаметра труб и ограничений по габаритам и соотношениям сторон на массу регенератора. 8 
прохода теплоносителей. Вследствие этого (рис. 7) масса регенератора при увеличении количества ходов сначала резко уменьшается, а потом начинает постепенно возрастать. При $\varepsilon=0,8-0,85$ количество ходов в гладкотрубном регенераторе рекомендуется принимать $n_{x}=4-8$ и $n_{x}=6-10$ при увеличении $\varepsilon$ до 0,9 и выше. Изменение потерь давления на этот диапазон не влияет.

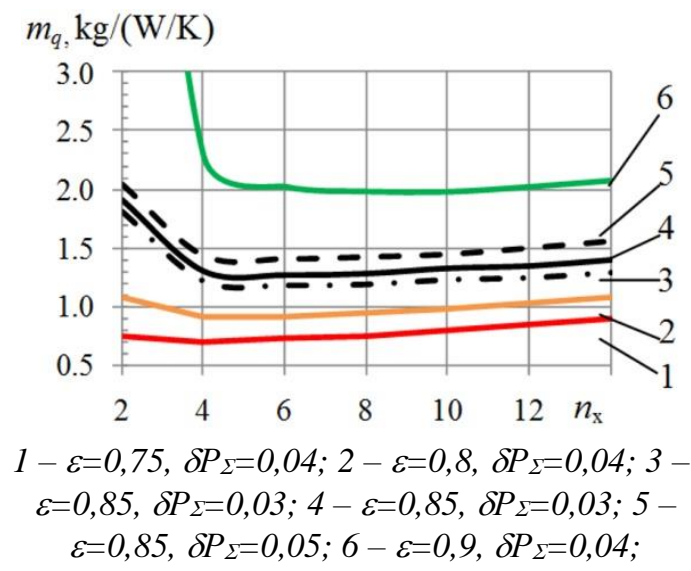

Рис. 7. Влияние количества ходов воздуха на относительную массу гладкотрубного змеевикового регенератора. ${ }^{9}$

Уменьшение относительных продольного и поперечного шагов на 0,1 при постоянных $\varepsilon, \delta P_{\text {hot }}, \delta P_{\text {cold }}$ приводит к уменьшению массы регенератора на $5 . . .6 \%$ (рис. 8 , непрерывные линии). Если же при проектировании регенератора допустить изменение величин $\delta P_{h o t}$, $\delta P_{\text {cold }}$ при постоянном значении суммарных потерь давления, то можно добиться уменьшение его массы в 1,4 раза (рис. 8).

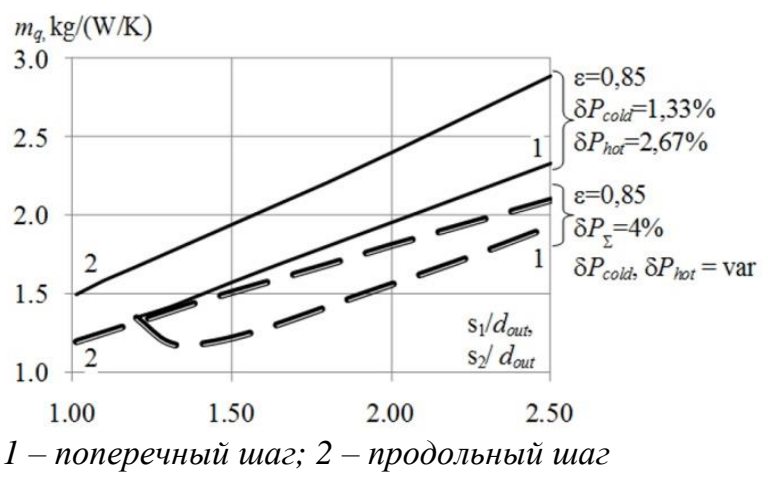

Рис. 8 Влияние шагов труб на относительную массу гладкотрубного змеевикового регенераTopa. $^{10}$

Минимум массы регенератора приходится на $s_{l} / d_{\text {out }}=1,25-1,38$. В качестве величины, характеризующей распределение давления между горячей и холодной сторонами регенератора, принято $k_{P}=\delta P_{\text {cold }} / \mathrm{d} P_{\Sigma}$.

Значение шагов труб в пучке ограничиваются:

- минимальной величиной конструктивного зазора $\delta_{\min }$ между поверхностью труб (1...2 мм):

- для поперечного шага $s_{1}-d_{\text {out }} \geq \delta_{\text {min }}$

- для продольного шага $s_{2}-d_{\text {out }} \geq \delta_{\text {min }}$

- для диагонального шага (шахматные пучки)

$$
\sqrt{\left(s_{1} / 2\right)^{2}+s_{2}^{2}}-d_{\text {out }} \geq \delta_{\min }
$$

- размерами дистанционных элементов (колец, пластин), которые придают пучку жесткость;

- размерами калачей и их взаимным расположением;

- радиусом сгиба труб;

- расстоянием между отверстиями в коллекторах, которое определяется из условия прочности коллектора.

Большой радиус гиба труб ограничивает минимальную величину продольного шага трубок, что приводит к увеличению массы и габаритов. При уменьшении радиуса гиба увеличивается сопротивление при движении воздуха между ходами, ухудшается технологичность производства конструкции, уменьшается толщина стенок труб в месте изгиба. Из технологических соображений (возможности изготовления калачей) минимальная величина радиуса гиба трубок была принята $R_{\text {bend }} / d_{\text {out }}=1$, что соответствует величине $s_{2} / d_{\text {out }}=1,5$.

Значительно на величину шагов (а, следовательно, и на массу) влияют условия прочности трубных досок или коллекторов. Их толщина существенно увеличивается при уменьшении шагов отверстий под трубы, поскольку малые шаги снижают прочность коллекторов и затрудняют закрепление в них труб (рис. 9). При закреплении в коллекторах диаметром 700 мм труб с внешним диаметром 22 мм и шагами равными шагам в пучке $\left(s_{1}=30\right.$ мм и $\left.s_{2}=22 \mathrm{mм}\right)$ толщина коллекторов при ресурсе 100 тыс. часов будет 14 мм, а суммарная масса коллекторов составит $29,6 \%$ от массы регенератора. Таким образом, размещение отверстий в коллекторе с шагами, равными шагам трубок в пучке, приводит к существенному увеличению массы. 


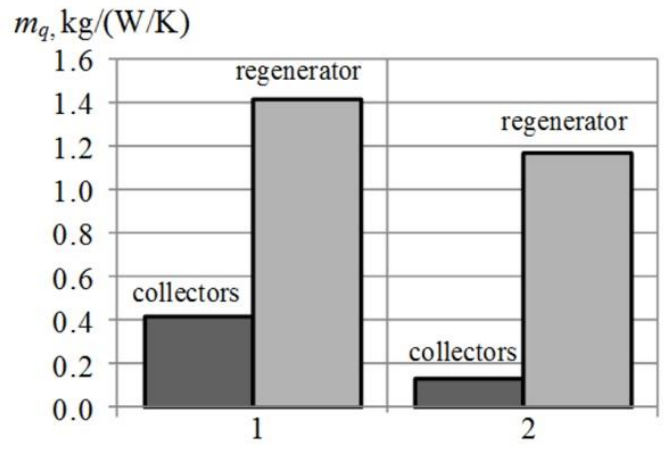

1 - непосредственное подсоединение труб к коллектору (шаг отверстий в коллекторе такой же, как и шаг труб); 2 - подсоединение труб к коллектору с помощью гнутых отводов (шаг отверстий в коллекторе увеличен в два раза).

Рис. 9. Массовые характеристики регенератора при разных способах подсоединения труб к коллекторам. ${ }^{11}$

С целью уменьшения массы коллекторов подвод труб к ним был выполнен с помощью гнутых отводов с увеличением продольного шага в 2 раза, что стало особенностью конструкции регенератора [34]. В результате толщина коллектора снизилась до 4,5 мм, масса коллекторов уменьшилась в 3,2 раза, а масса регенератора - на $22 \%$ (рис. 9).

\section{ОБСУЖДЕНИЕ}

Основную сложность при разработке регенеративных ГТУ представляет создание высокоэффективного и надежного регенератора, который имел бы приемлемые массогабаритные и стоимостные показатели.

Проектирование регенератора является многоцелевым оптимизационным процессом по поиску компромиссного решения между высокой тепловой эффективностью, низкими потерями давления, малыми массогабарит-

\section{APPENDIX 1 (ПРИЛОЖЕНИЕ 1)}

${ }^{1}$ Fig. 1. Packaging of 16-MW Gas Turbine Plant with heat regeneration ( 1 - gas turbine package; 2 - gas duct; 3 - regenerator; 4 - air ducts.).

${ }^{2,3}$ Table 1. Main design parameters of $16-\mathrm{mW}$ Gas Turbine Plant for the drive of supercharger of natural gas.

${ }^{4}$ Fig. 2. Smooth-pipe coil regenerator (1 - heat exchange matrix made of flat coil pipes; 2 - return bends; 3 - case; 4 - distance spacer; 5 - beams; 6 bent connectors; 7 - distributing air collector; 8 gathering air collector; 9 - displacer).

${ }^{5}$ Fig. 3. Geometrical characteristics of Gas Turbine smooth-pipe coil regenerator ( 1 - heat exchange matrix made of flat coil pipes; 2 - return bends; 3 - case; 4 - bent connectors; 5 - gathering air collector; 6 distributing air collector). ными показателями и низкой стоимостью. Эту проблему можно решить за счет выбора рациональных значений геометрических параметров теплообменной матрицы.

Выбор рациональных значений геометрических параметров теплообменных матриц регенератора необходимо проводить из условия минимальной массы всего теплообменника, а не только минимальной массы или объема его теплообменной матрицы, поскольку элементы конструкции, которые не принимают участие в процессах передачи тела существенно влияют на массу регенератора.

При проектировании необходимо учитывать ограничения, которые накладываются на геометрические параметры регенератора (размеры и шаги труб, габариты и форма теплообменника) исходя из условий технологичности, размещения на объекте и транспортирования. Указанные факторы не только приводят к росту массы, но и влияют на зависимость массовых характеристик теплообменника от геометрических параметров его теплообменной поверхности.

Изменение соотношения между величинами относительных потерь давления холодного и горячего теплоносителей (при постоянной величине $\delta P_{\Sigma}$ ) не оказывает значительного влияния на КПД, но существенно влияет на массогабаритные показатели регенератора. Выбором оптимального значения этой величины можно добиться значительного снижения массы регенератора.

Полученные результаты могут быть полезны при проектировании теплообменного оборудования для энергетических установок различных типов.

${ }^{6}$ Fig. 4. Dependence of mass and dimensional characteristics of regenerator on tubes diameters (other geometrical parameters of heat exchange matrix are invariable) (1 - specific mass of regenerator; 2 specific mass of heat exchange matrix; 3 - specific volume of heat exchange matrix; 3 - specific mass of heat exchange matrix; 4 - specific area of matrix surface; 5 - ratio between mass of heat exchange matrix and mass of regenerator ).

${ }^{7}$ Fig. 5. The impact of tubes diameter on mass and dimensions of smooth-pipe coil regenerator $(1-$ specific mass of regenerator $\left(m_{q}\right) ; 2$ - length $\left(L_{r e g}\right) ; 3$ width $\left(B_{\text {reg }}\right) ; 4$ - height $\left.\left(H_{\text {reg }}\right)\right)$.

${ }^{8}$ Fig. 6. The impact of tube overall dimensions limitation on mass of regenerator ( 1 - computation without overall dimensions limitation; 2 - computation with width limitation $\left(B_{r e g}\right) ; 3$ - computation with length limitation $\left(L_{r e g}\right) ; 4-$ computation with strict 
requirements to form of regenerator cross section $L_{r e g} / B_{r e g}=\left(L_{r e g} / B_{r e g}\right)_{g i v} ; 5-$ computation with 0.7 $\left(L_{r e g} / B_{r e g}\right)_{g i v} \leq L_{r e g} / B_{r e g} \leq 1.3\left(L_{r e g} / B_{r e g}\right)_{g i v}$.

${ }^{9}$ Fig. 7. The impact of number of air motions on specific mass of tubular coil pipes regenerator.

${ }^{10}$ Fig. 8. The impact of tube steps on specific mass of tubular coil pipes regenerator $(1$ - cross step; 2 longitudinal step).

\section{Литература (References)}

[1] Meherwan P. Boyce, Gas Turbine Engineering Handbook. 4th ed. Waltham, Elsevier Inc., 2012, $1169 \mathrm{p}$.

[2] Gas turbine world 2019 GTW handbook, vol. 34., Fairfield, Pequot Publishing Inc., 2019, 154 p.

[3] Romanov V. I. (On ed.) Nikolaevskie gazoturbinnye dvigateli i ustanovki [Nikolaev`s Gas Turbine Engines and Plants]. Nikolaev, Yug-Inform, 2005, 304 p. (In Russian).

[4] Zaryankin A. E., Grigoryev E. Yu., Buzulutskii D. E., Hazov P. S., O vozmozhnykh putyakh povysheniya moshchnosti, nadezhnosti i ekonomichnosti gazoturbinnykh ustanovok [Possible Ways of Improving Capacity, Reliability and Economic Efficiency of Gas Turbines]. "Vestnik IGEU" [Proc. of the ISEU], 2014, issue 4, pp. 1 - 10. (In Russian).

[5] McDonald C. F., The Increasing Role of Heat Exchangers in Gas Turbine Plant. ASME paper, ASME, New York, 1989, no. 89-GT-103, 12 p.

[6] Anoshkin I. S., Pohodyaev S.B., Yalin V. A., Teploobmennoe oborudovanie XXI veka dlya GTD novogo pokoleniya [Heat Exchange Equipment of XXI Century for Gas Turbine Engine of New Generation]. Gazoturbinnye tekhnologii -Gas Turbine Technologies, 2006, no. 5, p. 22-23. (In Russian).

[7] McDonald C. F., Wilson D. G., The Utilization of Recuperated and Regenerated Engine Cycles for High Efficiency Gas Turbines in the 21st Century. Applied Thermal Engineering, 1996, vol. 16., issue 8-9, pp. $635-653$.

[8] Weiler C. L., WR-21 Design and Maintenance. ASME paper, ASME, New York, 1996, no 96GT-328, 25 p.

[9] David T. Mercury ${ }^{\mathrm{TM}} 50$ Field Evaluation and Product Introduction. Industrial Application of Gas Turbines Committee, 2005, 10 p.

[10] McDonald C. F., Low-Cost Compact Primary Surface Recuperator Concept for Microturbines. Applied Thermal Engineering, 2000, vol. 20, issue 5, pp. $471-497$.

[11] Paton B., Khalatov A., Kostenko D., Bileka B., Pis'mennyi E., Botsula A., Parafiynyk V, Konyakhin V. Kontseptsiya (proekt) derzhavnoi naukovo-tekhnichnoi programy "Stvorennya promyslovykh gazoturbinnykh dvyguniv novogo pokolinnya dlya gazovoyi promyslovosti ta energetyky" [Concept (draft) of the State Scientific and Technical Programs "Development of Indus-
${ }^{11}$ Fig. 9. Mass characteristics of regenerator with different methods of attachment tubes to collectors (1 - direct attachment of tubes to collectors (step of holes in collector is the same as the step of tubes); 2 attachment of tubes to collectors via bent connectors (step of holes in collector is increased by two times)).

trial Gas Turbine Engines of a New Generation for Gas Industry and Power Engineering"]. "Vestnik NAN Ukrainy" [Proc. of the NAS of Ukraine], 2008, no. 4. pp. 3-9 (In Ukrainian).

[12] Belousov V.D., Danilenko V.G., Mishustin N.I., Rybakov V. P., Trubchatye regeneratory OAO "ZiO-Podol'sk" [Tubular regenerators by JSC "ZiO-Podol'sk"]. Gazoturbinnye Tekhnologii Gas Turbine Technologies, 2004, no. 5, pp. 4445. (In Russian).

[13] Memona A. G., Harijan K., Uqaili M. A., Memon R. A., Thermo-Environmental and Economic Analysis of Simple and Regenerative Gas Turbine Cycles with Regression Modeling and Optimization. Energy Conversion and Management, 2013, vol. 76, pp. 852-864.

[14] Chen L. Lia Y., Suna F., Wub C., Power Optimization of Open-Cycle Regenerator Gas-Turbine Power Plants. Applied Energy, 2004, vol. 78, issue 2 (June), pp. 199-218.

[15] Hanafizadeh P., Parhizgar T., Gheimasi A. N., Analysis of Micro-Recuperators in Small-Sized Gas Turbines-Manufacturing Potential of Iran. Energy Equipment and Systems, 2015, vol. 3, no. 1, pp. 1-12.

[16] Shah R. K., Compact Heat Exchangers for Microturbines. Proc. 5th Int. Conf. "Enhanced, Compact and Ultra-Copmact Heat Exchangers: Science, Engineering and Technology". NJ, 2005, pp. 247-257.

[17] Xiao G., Yang T., Liu H., Ni D., Ferrari M. L., Li M., Luo Z., Cen K., Ni M., Recuperators for Micro Gas Turbines: A review. Applied Energy, 2017, vol. 197, pp. 83-99.

[18] Alklaibi A. M., Khan M. N., Khan W. A., Thermodynamic Analysis of Gas Turbine with Air Bottoming Cycle. Energy, 2016, vol. 107 (July), pp. 603-611.

[19] Nkoi B., Pilidis P., Nikolaidis T., Performance Assessment of Simple and Modified Cycle Turboshaft Gas Turbines. Propulsion and Power Research, 2013, vol. 2, issue 2, pp. 96-106.

[20] Brighenti G. D., Orts-Gonzalez P. L., Sanchezde-Leon L., Zachos P. K. Design Point Performance and Optimization of Humid Air Turbine Power Plants. Applied Sciences, 2017, № 7, pp. 413-435.

[21] Rovira A., Analysis and Optimization of Combined Cycles Gas Turbines Working with Partial Recuperation. Energy Conversion and Management, 2015, vol. 106, pp. 1097-1108. 
[22] Ruixian C., Lixia J., Analysis of the Recuperative Gas Turbine Cycle with a Recuperator Located between Turbines. Applied Thermal Engineering, 2006, vol. 26, issue 1, pp. 89-96.

[23] Antuf'ev V.M., Gysev E. K., Ivakhnenko V. V., Kuznetsov E. F., Lamm Ya. A. Teploobmennye apparaty iz profil nykh listov [Heat Exchangers from Channeled Sheet]. Leningrad, Energiya, 1972, 127 p. (In Russian).

[24] Traverso A., Zanzarsi F., Massardo A. Cheope A., Tool for the Optimal Design of Compact Recuperators. Proc. ASME Turbo Expo 2004: Power for Land, Sea, and Air, 2004, no. GT200454114. pp. 115-123.

[25] Bahman Z., Compact Heat Exchangers. Selection Application Design and Evaluation. Springer International Publishing, 2017, pp. 559.

[26] Campbell J. F., Rohsenow W. M., Gas Turbine Regenerators: A Method for Selecting the Optimum Plate-Finned Surface Pair for Minimum Core Volume. International Journal of Heat and Mass Transfer, 1992, vol. 35, issue 12 (December), pp. 3441-3450.

[27] Sayyaadi H., Aminian H.,, Design and Optimization of a non-TEMA Type Tubular Recuperative Heat Exchanger Used in a Regenerative Gas Turbine, Energy, 2010, vol. 35, issue 4, pp. 16471657.

[28] Durmus A, Benli H, Kurtbas I, Gül H., Investigation of Heat Transfer and Pressure Drop in Plate Heat Exchangers Having Different Surface Profiles. International Journal of Heat Mass Tranfer, 2009, vol. 52, issues 5-6, pages 14511457.
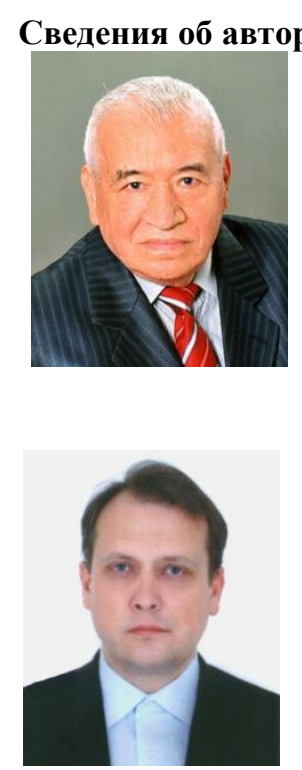

Горбов Виктор Михайлович, к.т.н., профессор, заведующий кафедрой ЭСЕУ и Т НУК им. адмирала Макарова. Область научных интересов: судовые энергетические установки с газотурбинными двигателями. E-mail:

viktor.gorbov@nuos.edu.ua

Соломонюк Денис Николаевич, к.т.н., ведущий инженерконструктор ГП НПКГ "Зоря""Машпроект", ассистент кафедры ЭСЕУ и Т НУК им. адмирала Макарова. Область научных интересов: утилизационные котельные установки и теплообменные аппараты ГТУ. E-mail: denys.solomoniuk@nuos.edu.ua
[29] Song Y., Asadi M., Xie G., Rocha. L.A.O. Constructive Wavy-Fin Channels of a Compact Heat Exchanger with Heat Transfer Rate Maximization and Pressure Losses Minimization. Applied Thermal Engineering, vol. 75, pp. 24-32.

[30] Guo J., Huai X., Optimization Design of Recuperator in a Chemical Heat Pump System Based on Entrance Dissipation Theory. Energy, vol. 41, issue 1, pp. 335-343.

[31] Tikhonov A. M., Regeneratsiya tepla v aviatsionnykh GTD [Heat Regeneration in Aircraft Gas Turbine Engine] Moscow, Mashynostroenie, 1977, 107 p. (In Russian).

[32] Kays W. M., London A. L., Compact Heat Exchangers, New York: McGraw-Hill Comp., 1984, 224 p.

[33] Bazhan P. I., Kanevts G. E., Seliverstov V. M. Spravochnik po teploobmennym apparatam. [Heat Exchanges Handbook] Moscow, Mashynostroenie, 1989, 365 p. (In Russian).

[34] Bezdelova O. N., Solomonyuk D. N., Movchan S.M., Chobenko V. M., Bochkaryov Yu. V., Regenerator gasoturbinnoy ustanovki [Regenerator of Gas-Turbine Unit]. Patent UA, no. 78601, 2013. (In Ukranian).

[35] Attetkov A. V., Zarubin V. S., Kanatnikov A. N., Metody optimizatsii [Optimisation Methods]. Moscow, Infra-M, 2013, 272 p. (In Russian).

[36] Baklastov A. M., Gorbenko V. A., Udyma P. G., Proektirovanie, montazh i ekspluatatsiya teplomassobmennykh ustanovok [Design, Installation and Operation of Heat and Mass Transfer Plants], Moscow, Energoatomizdat, 1981, 336 p. (In Russian).

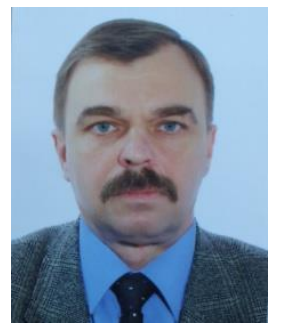

Мовчан Сергей Николаевич, начальник отдела котлов и теплообменных аппаратов ГП НПКГ "Зоря"-"Машпроект". Область научных интересов: утилизационные котельные установки и теплообменные аппараты ГТУ. E-mail: movchan.serg64@gmail.com 